\title{
Potential role of Nigella sativa supplementation with physical activity in prophylaxis and treatment of COVID-19: a contemporary review
}

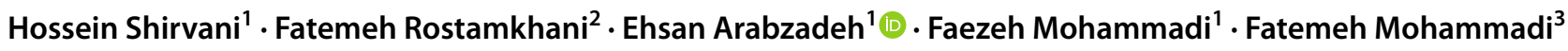

Received: 9 February 2021 / Accepted: 18 May 2021 / Published online: 28 May 2021

(c) The Author(s), under exclusive licence to Springer-Verlag Italia S.r.l., part of Springer Nature 2021

\begin{abstract}
The widespread prevalence and mortality of coronavirus diseases-2019 (COVID-19) lead many researchers to study the SARS-CoV-s2 infection to find a treatment for this disease. Discovering the mechanisms of action of COVID-19 and coping at the cellular level with this disease can have better effects. Including the target tissues of this disease are the lungs and the immune system. It is stated that COVID-19 easily infiltrates into alveoli through its receptors and then starts to proliferate. Subsequently, with the weakening of immune cells and increase inflammatory cytokines, it increases the rate of inflammation in the body. Strengthening the immune system and inhibiting COVID-19 receptors can play a preventive or even therapeutic role for this disease. Nigella sativa ( N. sativa) is one of the herbal medicines to possess numerous pharmacological effects related to several organs of the body. Among the extraordinary properties of this plant is improving asthma and several lung diseases. The recent studies have shown that $N$. sativa at the cellular level can inhibit COVID-19 receptors. It was also stated that performing regular exercise training (especially moderate-intensity exercise training) can modulate the immune system and have an anti-inflammatory effect. Since the use of herbal supplements with exercise can have tremendous therapeutic effects at the cellular level, the hypothesis to use the Nigella sativa along with exercise training to prophylaxis and treatment COVID-19 will be highlighted in this paper.
\end{abstract}

Keywords COVID-19 $\cdot$ Nigella sativa supplementation $\cdot$ Physical activity $\cdot$ Exercise $\cdot$ Treatment $\cdot$ Prevention

\section{Introduction}

COVID-19 has been recognized as pandemic by the world health organization (WHO) since March 11, 2020 [1]. It has been shown that COVID-19 causes wide mortality and has also irreparable economic damage to countries around the world. COVID-19 initially affects the cells of the small air sacs known as the alveoli. Alveoli are made up of alveolar cells (also known as pneumocytes) and alveolar macrophages. COVID-19 causes inflammation in the lung tissue, which is usually accompanied by symptoms such as dry

Ehsan Arabzadeh

eh.arabzadeh@bmsu.ac.ir

1 Exercise Physiology Research Center, Life Style Institute, Baqiyatallah University of Medical Sciences, Tehran, Iran

2 Department of Biology, College of Basic Sciences, Yadegar-E-Imam Khomeini (RAH) Shahre Rey Branch, Islamic Azad University, Tehran, Iran

3 Maternal, Fetal\& Neonatal Research Center, Tehran University of Medical Sciences (TUMS), Tehran, Iran cough, chest pain, fever, and difficulty breathing [2]. These symptoms gradually worsen and cause death in people with COVID-19 [3].

Simultaneously with the involvement of lung tissue, the immune system usually reacts to fight the virus. It is stated that in COVID-19, changes in patients' immune systems are more similar. Usually, after COVID-19, a decrease in the number of CD4+ and CD8+ lymphocytes, monocytes and platelets has been recorded with an increase in the number of neutrophils [4]. Elevated concentrations of CRP, interleukin-6 (IL-6), serum ferritin, and erythrocyte sedimentation rate (ESR) have been reported in COVID-19 patients [5]. In addition to these factors, an excessive increase in some cytokines, such as IL-7, IL-10, GCSF, IP10, MCP1, MIP1A, and TNF- $\alpha$ has been observed [6, 7]. In contrast, an increase in lymphocyte levels was observed in the review of the improved immune system [5]. Therefore, strengthening and improving the function of lung cells, strengthening the immune system, and controlling pro-inflammatory cytokines can be suitable for the prevention or even treatment of COVID-19. There are lots of research that has been 
done on COVID-19, and they have proposed various treatment strategies. Meanwhile, exercise physiology scientists, especially molecular and cellular exercise physiologists, also use various exercise intensities as a preventive method for COVID-19 due to the positive effects of exercise training on the immune system [8-10].

Because the use of herbal supplements improves the effects of regular exercise training (reducing ROS), it seems that the combination of exercise training and herbal supplement can counteract COVID-19 or even reduce destructive effects of COVID-19 [11-13]. Nigella sativa ( $N$. sativa) is one of the useful herbal medicines that have a strong positive effect on the lungs and also improve the immune system. Therefore, in this study, the hypothesis considers the potential role of $N$. sativa supplementation with physical activity in the prophylaxis and treatment of COVID-19.

\section{Effectiveness of Nigella sativa on lung tissue, immune system, and COVID-19}

Nigella sativa, known as black cumin belongs to the Ranunculaceae family. $N$. sativa has been used all over the world in cooking and for its many medicinal properties. Various studies have confirmed the beneficial effects of $N$. sativa on the respiratory system, lung parenchyma, narrowing of the airways, stimulating the body's energy, and improving fatigue, as well as its anti-inflammatory effects [14-16]. The seeds of this plant are very useful and have different chemical compositions and also contain amino acids, proteins, carbohydrates, and volatile oil ( 0.5 to maximum $1.5 \%$ ), and fixed oil (30\%). Many of the medicinal properties of $N$. sativa are due to the quinone compounds in its seed [17]. The composition of this plant includes four types of alkaloids called Nigellamine (1) A, (2) A, (1) B, (2) B, which is extracted from the seed of this plant. Thymoquinone (TQ), Dithymoquinone (TQ2), Thymohydroquinone (THQ), and Thymol are the main active ingredients in the aqueous extract of the $N$. sativa [18] (Fig. 1).

Nigella sativa is useful for improving and treatment of respiratory disorders, such as asthma and bronchial spasm [19]. Nigellon is an N. sativa compound (a polymer in TQ or TQ2) that can relieve airway obstruction. In fact, Nigellone blocks the release of histamine, which causes narrowing of the airways [19]. Numerous studies have also investigated the anti-inflammatory [14] and anti-asthmatic [20] effects of $N$. sativa in lung tissue. Therefore, $N$. sativa can have therapeutic effects in most lung diseases [21].

Regarding the effect of $N$. sativa on the immune system, it has been shown that the distribution of $N$. sativa effects on pro-and anti-inflammatory cytokines (increasing anti-inflammatory cytokines and decreasing pro-inflammatory cytokines). Also, N. sativa can modulate Th1/Th2<smiles>Cc1ccc(C(C)C)c(O)c1</smiles>

(a)<smiles>CC1=CC(=O)C(C(C)C)=CC1=O</smiles>

(b)

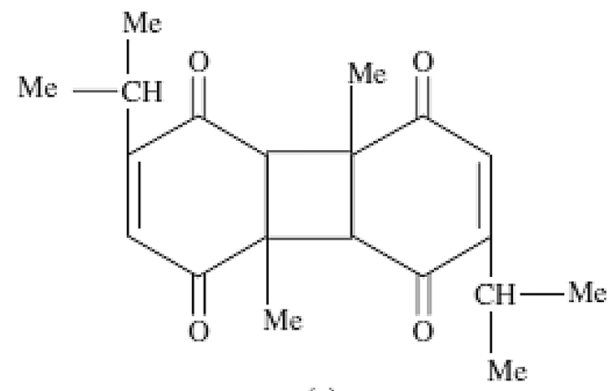

(c)

Fig. 1 Chemical structure of Thymol (a), Thymoquinone (b), and Dithymoquinone (c)

lymphocytes function [22]. Khabbazi et al. showed the immunomodulatory effects of $N$. sativa on T lymphocytes in chronic disease and stated that treatment with $N$. sativa improves rheumatoid arthritis through its immunomodulatory effects on T cells [23]. It is stated that $N$. sativa is able to activate $\mathrm{T}$ lymphocytes and have an immunomodulatory effect in human subjects [24]. Oral gavage of N. sativa in diabetic animals, increases lymphocyte content in the peripheral bloodstream [25]. Owing to the beneficial effects of $N$. sativa on the lung tissue and immune system, it seems $N$. sativa may also play a treatment role in COVID-19.

It has been shown that TQ has the ability to block ACE2 (a COVID-19 receptor) [26]. In other words, TQ may block the entry of COVID-19 into pneumocytes. Therefore, both nigellimine and thymoquinone from $N$. sativa may be considered as potentially active pharmacological components for the treatment of COVID-19. Recently, Rahman showed that $N$. sativa, with a wide range of bioactive components, such as nigellimine and thymoquinone, may have many benefits in the treatment of COVID-19, including prevention of the virus enters the pneumocyte and prepares an ionophore for an enhanced uptake of $\mathrm{Zn}^{2+}$, which can increase the host's immune response to COVID-19 and also prevent viral replication by blocking viral RdRp. This researcher suggested a dose of black seed oil between 40 and $80 \mathrm{mg} /$ $\mathrm{kg}$ per day as adjunctive therapy without any side effects [26]. Ulasli et al. showed that $N$. sativa reduces the coronavirus load in infected HeLa cells by stimulating IL- 8 secretion and downregulation of expression of transient receptor 
potential (TRP) genes, such as TRPM6, TRPA1, TRPC4, and TRPM7 [27]. Also, it has been shown that N. sativa components or antiviral drugs can limit COVID-19 targets, such as main proteases (6LU7 and 6Y2E), main peptidase (2GTB), angiotensin-converting enzyme 2 (ACE2), and heat shock protein A5 [28].

Some natural compounds may prevent the coronavirus from adhering to the host epithelial cell. It has been shown that Nigelledine, an N. sativa alkaloid, can block 6LU7, and $\alpha$-Hederin, a saponin of $N$. sativa, can block 2GTB [28]. As stated TQ is also one of the essential oils of $N$. sativa that tends to bind to 6LU7, ACE2, and heat shock protein A5 active sites [29]. Also, Hederagenin (a saponin in $N$. sativa) binds with 6LU7, 6Y2E, ACE2, and active sites of GRP78 [30]. Thymohydroquinone showed moderate binding energy to SARS-CoV-2 6LU7, endoribonuclease, ADPribose-1-phosphatase, RNA-dependent RNA polymerase, SARS-CoV-2 protein-binding domain [31]. Nigellidine also showed high binding to COVID-19 enzymes and proteins, such as N-terminus-proteinase, 6LU7, nonstructural protein 2 , spike-glycoprotein, and nucleocapsid. Nigellidine has a high affinity for inflammatory signal molecules and other proteins, such as human IL1R (1itb), TNFR1 (1ncf), and TNFR2 (3alq) [32]. According to this research, some components of $N$. sativa provide the ability to inhibit the entry of coronavirus into lung cells and immunity, including nigellidine, $\alpha$-hederin, hederagenin, thymohydroquinone, and thymoquinone.

\section{Effectiveness of physical activity on lung tissue, immune system, and COVID-19}

In the recent decades, exercise and physical activity have been considered appropriate treatment options for respiratory and lung diseases [33]. Exercise can increase functional capacity, increase exercise tolerance, and improve the health-related quality of life (HRQoL) in lung disease $[33,34]$. Fatigue is one of the most common symptoms in patients with respiratory disorders [35]. Keyser et al. showed that aerobic exercise reduces the rate of fatigue in interstitial pulmonary disease (ILD) [36]. Decreased fatigue was associated with an improvement in the patient's perception of fatigue and increased exercise capacity [36]. Casaburi et al. also showed that in obstructive pulmonary disease, aerobic exercise (8-week, 90\% aerobic threshold on cycle ergometer) reduces lactate acidosis and improves ventilation [37]. It has been shown that low and moderate intensity aerobic exercise (50 and $75 \%$ of maximal speed) reduces airway inflammation and improves lung remodeling in asthmatic mice [38]. Aerobic exercise also reduces parenchymal artery inflammation. These effects of exercise on lung tissue may be due to a decrease in the Th2 response, an increase in interleukin-10, and a decrease in the expression of NF-KB, MCP-1, and IGF-I [39]. It has been suggested that part of the effects of exercise on the immune system is due to the ability of exercise to modulate the cellular metabolism of lymphocytes, especially glucose and glutamine. It was observed that the change in lymphocyte function is associated with different effects of moderate exercise intensity $\left(40-60 \% V_{2} \mathrm{O}_{\max }\right)$ on $\mathrm{T}$ and B lymphocyte metabolism [40]. In particular, T lymphocytes increase glutamine intake by altering the metabolism of this amino acid to the aerobic pathway. At the same time, these cells reduce glucose intake and lactate production levels. In contrast, B lymphocytes showed that it increases the consumption of both glucose and glutamine [40]. All of these changes in lymphocytes can provide a stronger defense of lymphocytes against viruses. Owing to the wide cellular effects of exercise training on lung tissue, immune system, and viral diseases, it seems that it could be used to prevent viral diseases, especially COVID-19. Few studies have examined the preventive and even therapeutic effects of exercise on COVID-19.

As mentioned, one of the recognizable features in COVID-19 disease is cytokine storm which includes increase: IL-6, IL-1B, IL-2, IL-8, IL-17, G-CSF, GM-CSF, IFN- $\gamma$, IP10, MCP1, MIP1a, and TNF- $\alpha$, and is known as an acute systemic inflammatory syndrome [41], which can induce oxidative stress, which is detrimental to cells. Among the effects of exercise training, especially aerobic exercise training (40 min each session, 3 times per week for 6 months; first 3 months $=60-70 \%$ of HRmax, second 3 months $=70-80 \%$ of HRma) is the strengthening of antiinflammatory cytokines and the improvement of oxidative stress in elderly [42]. Falkenberg et al. in a systematic review study examined the effect of yoga mental exercise on immune function. Their results showed that yoga reduced levels of TNF- $\alpha$, IL- 6, IL- $1 \beta$ as well as improved cellular and mucosal immunity in people who were suffering from inflammatory diseases [43]. An acute exercise session (moderate intensity less than $1 \mathrm{~h}$ ) has been shown to activate immune system cells in the bloodstream and various tissues (such as mucosal surfaces including respiratory and gut epithelial), which strengthens the innate immune system [44]. Regular aerobic exercise (moderate intensity) also increases the antipathogenic activity of macrophages, which is associated with increased blood flow immunoglobulins, neutrophils, anti-inflammatory cytokines, immature B cells, the dramatic influx of natural killer cells (NK), and CD8 + T cells $[45,46]$. Also, acute and chronic moderate-intensity exercise regulates excessive inflammation in the airways [47]. Chronic exercise (over a 6-month period) prevents age-related immune dysfunction (chronic inflammation) and improves the effectiveness of influenza vaccination in the elderly without harmful side effects [48]. The effect of moderate exercise (chronic, $40-60 \% V_{2} \mathrm{O}_{\max }$ ) on immune 
function is especially beneficial for the elderly because they are vulnerable to COVID-19. There is evidence that regular physical activity and an active lifestyle are beneficial for immune function [49]. Exercise training has been known as a lifestyle factor that can maintain and even promote immune function and act like a vaccine against certain diseases/infections through producing physiological stress in the human body which lead to a series of adaptations occurring to overcome these stimuli [50].

Based on the clinical studies in humans, performing short-term exercise (e.g. 45-60 min), and moderate exercise $\left(40-60 \% V_{2} \mathrm{O}_{2 \max }\right)$, at least 3 times per week is useful for the immune system [51]. Moderate-intensity exercise is also associated with increased leukocyte function in humans [52]. Exercise increases chemotaxis, degranulation, cytotoxic activity, phagocytosis, and oxidative activity of neutrophils and macrophages in rats [53]. Increased cytolytic activity of NK cells and activating lymphokine-activated killer cells (LAK) during 60 min of moderate-intensity exercise has also been reported by healthy cyclists [54]. Various studies have shown that regular exercise (resistance or endurance training) increases macrophage phagocytosis, oxidative burst, neutrophil oxidative burst, percentage of TCD4 lymphocytes, and reduces circulating TNF- $\alpha$ and IL- 6 [51]. Therefore, exercise training with positive effects on the immune system can play a therapeutic and preventive effect against COVID-19.

\section{Potential role of Nigella sativa supplementation with physical activity against COVID-19}

Lung tissue is one of the most important issues that are responsible for providing oxygen for oxidative phosphorylation during daily physical activity and exercise training. Exercise training and regular physical activity can strengthen the alveoli as well as the immune system associated with improving lung cells and ultimately functional capacity [38, $55,56]$. On the other hand, in traditional medicine, special attention has been given to the use of $N$. sativa in the treatment of pulmonary diseases. Most of these studies have given attention to the medicinal properties of this plant on the respiratory system $[20,57]$. However, no study has been performed on the use of $N$. sativa along with exercise training on COVID-19. Many common mechanisms of exercise training and $N$. sativa can affect COVID-19. It seems that consumption of black seed (black seed oil dose $40-80 \mathrm{mg} /$ $\mathrm{kg} /$ day) along with regular aerobic exercise (40-60\% $V \mathrm{O}_{2 \max }$ ) could strengthen the immune system by increasing lymphocyte activity and metabolism, reducing cytokine storms, increasing anti-inflammatory cytokines, and inhibiting COVID-19 receptors (by various active components of
$N$. sativa including TQ), which may play a prophylaxis and treatment role for COVID-19.

\section{Conclusion}

The recent studies have shown that $N$. sativa can play a therapeutic role for COVID-19 by strengthening the immune and respiratory systems as well as inhibiting Covid-19 receptors. On the other hand, exercise training can have anti-inflammatory effects and improve immunity. Also, studies show that low to moderate intensity exercise training is useful for some respiratory diseases. Based on the previous research, it seems that a lifestyle combined with exercise training and $N$. sativa supplementation can be effective in reducing COVID19 mortality.

Funding None. The authors do not have any personal or institutional financial interests in drugs, materials, or devices described in this paper.

\section{Declarations}

Conflict of interest The authors declare that they have no known competing financial interests or personal relationships that could have appeared to influence the work reported in this paper.

Informed consent Not applicable.

Ethical approval This article does not contain any studies with human participants performed by any of the authors.

\section{References}

1. Cucinotta D, Vanelli M (2020) WHO declares COVID-19 a pandemic. Acta Bio Medica Atenei Parmensis 91(1):157-163

2. Huang C, Wei Y, Wu W, Xie X, Yin W, Li H, Liu M, Xiao Y, Gao H, Guo L, Xie J, Wang G, Jiang R, Gao Z, Jin Q, Wang J, Cao B et al (2020) Lancet 395(10223):497-506

3. Mallapaty S (2020) Scientists fear coronavirus spread in vulnerable nations. Nature 578(7795):348-348

4. Wang L et al (2020) Coronavirus disease 2019 in elderly patients: characteristics and prognostic factors based on 4-week follow-up. J Infect 80:639-645

5. Chen $\mathrm{N}$ et al (2020) Epidemiological and clinical characteristics of 99 cases of 2019 novel coronavirus pneumonia in Wuhan, China: a descriptive study. Lancet Lond Engl 395:507-513

6. Lescure F-X et al (2020) Clinical and virological data of the first cases of COVID-19 in Europe: a case series. Lancet Infect Dis 20:697-706

7. Wang Z et al (2020) Clinical features of 69 cases with coronavirus disease 2019 in Wuhan, China. Clin Infect Dis 37:420-430

8. Scudiero $\mathrm{O}$ et al (2021) Exercise, immune system, nutrition, respiratory and cardiovascular diseases during COVID-19: a complex combination. Int J Environ Res Public Health 18(3):904 
9. da Silveira MP et al (2020) Physical exercise as a tool to help the immune system against COVID-19: an integrative review of the current literature. Clin Exp Med 21:1-14

10. Scartoni FR et al (2020) Physical exercise and immune system in the elderly: implications and importance in COVID-19 pandemic period. Front Psychol 11:3215

11. Sellami M et al (2018) Herbal medicine for sports: a review. J Int Soc Sports Nutr 15(1):1-14

12. Molanouri SM, Amani SS (2020) Exercise training, immune system, and coronavirus. Res Sport Sci 12(46):17-40

13. Chakraborty $S$ et al (2020) COVID-19 pandemic: pharmacological uses of plants to boost immune system. Res J Med Health Sci 1(1):17-21

14. Boskabady MH et al (2011) The effect of Nigella sativa alone, and in combination with dexamethasone, on tracheal muscle responsiveness and lung inflammation in sulfur mustard exposed guinea pigs. J Ethnopharmacol 137(2):1028-1034

15. Gholamnezhad Z et al (2019) Clinical and experimental effects of Nigella sativa and its constituents on respiratory and allergic disorders. Avicenna J Phytomed 9(3):195

16. Mokhtari-Zaer A et al (2020) The protective effect of Nigella sativa extract on lung inflammation and oxidative stress induced by lipopolysaccharide in rats. J Ethnopharmacol 253:112653

17. Gali-Muhtasib H, El-Najjar N, Schneider-Stock R (2006) The medicinal potential of black seed (Nigella sativa) and its components. Adv Phytomed 2:133-153

18. Venkatachallam SKT et al (2010) Chemical composition of Nigella sativa $\mathrm{L}$. seed extracts obtained by supercritical carbon dioxide. J Food Sci Technol 47(6):598-605

19. Gilani A-UH, Jabeen Q, Khan MAU (2004) A review of medicinal uses and pharmacological activities of Nigella sativa. Pak J Biol Sci 7(4):441-451

20. Khan MA (1999) Chemical composition and medicinal properties of Nigella sativa Linn. Inflammopharmacology 7(1):15-35

21. Kanter M (2009) Effects of Nigella sativa seed extract on ameliorating lung tissue damage in rats after experimental pulmonary aspirations. Acta Histochem 111(5):393-403

22. Majdalawieh AF, Hmaidan R, Carr RI (2010) Nigella sativa modulates splenocyte proliferation, Th1/Th2 cytokine profile, macrophage function and NK anti-tumor activity. J Ethnopharmacol 131(2):268-275

23. Khabbazi A et al (2020) A systematic review of the potential effects of Nigella sativa on rheumatoid arthritis. Planta Med 86(07):457-469

24. Kheirouri S, Hadi V, Alizadeh M (2016) Immunomodulatory effect of Nigella sativa oil on T lymphocytes in patients with rheumatoid arthritis. Immunol Invest 45(4):271-283

25. Fararh K et al (2004) Mechanisms of the hypoglycaemic and immunopotentiating effects of Nigella sativa L. oil in streptozotocin-induced diabetic hamsters. Res Vet Sci 77(2):123-129

26. Rahman MT (2020) Potential benefits of combination of Nigella sativa and Zn supplements to treat COVID-19. J Herb Med 23:100382

27. Ulasli M et al (2014) The effects of Nigella sativa (Ns), Anthemis hyalina (Ah) and Citrus sinensis (Cs) extracts on the replication of coronavirus and the expression of TRP genes family. Mol Biol Rep 41(3): 1703-1711

28. Bouchentouf S, Missoum N (2020) Identification of compounds from Nigella sativa as new potential inhibitors of 2019 novel Coronavirus (Covid-19): molecular docking study. Med Chem 28(12):35-41

29. Ghosh $\mathrm{R}$ et al (2020) Evaluation of green tea polyphenols as novel corona virus (SARS CoV-2) main protease (Mpro) inhibitors-an in silico docking and molecular dynamics simulation study. J Biomol Struct Dyn. https://doi.org/10.1080/07391102.2020.1779818
30. Sampangi-Ramaiah MH, Vishwakarma R, Shaanker RU (2020) Molecular docking analysis of selected natural products from plants for inhibition of SARS-CoV-2 main protease. Curr Sci 118(7):1087-1092

31. da Silva JKR et al (2020) Essential oils as antiviral agents, potential of essential oils to treat SARS-CoV-2 infection: an in-silico investigation. Int J Mol Sci 21(10):3426

32. Maiti $\mathrm{S}$ et al (2020) Active-site molecular docking of Nigellidine to nucleocapsid/Nsp2/Nsp3/MPro of COVID-19 and to human IL1R and TNFR1/2 may stop viral-growth/cytokine-flood, and the drug source Nigella sativa (black cumin) seeds show potent antioxidant role in experimental rats. J Drug Target 27(19):245-251

33. Bade BC et al (2015) Increasing physical activity and exercise in lung cancer: reviewing safety, benefits, and application. J Thorac Oncol 10(6):861-871

34. Dowman LM et al (2015) Effect of disease aetiology on response to exercise training in patients with interstitial lung disease. B16. New randomised controlled trials in pulmonary rehabilitation. American Thoracic Society, pp A2460-A2460

35. Wilt TJ et al (2007) Management of stable chronic obstructive pulmonary disease: a systematic review for a clinical practice guideline. Ann Intern Med 147(9):639-653

36. Keyser RE et al (2015) Changes in fatigability following intense aerobic exercise training in patients with interstitial lung disease. Respir Med 109(4):517-525

37. Casaburi R et al (1991) Reductions in exercise lactic acidosis and ventilation as a result of exercise training in patients with obstructive lung disease. Am Rev Respir Dis 143(1):9-18

38. Vieira RP et al (2007) Aerobic exercise decreases chronic allergic lung inflammation and airway remodeling in mice. Am J Respir Crit Care Med 176(9):871-877

39. Vieira RP et al (2008) Aerobic conditioning and allergic pulmonary inflammation in mice. II. Effects on lung vascular and parenchymal inflammation and remodeling. Am J Physiol Lung Cell Mol Physiol 295(4):L670-L679

40. Navarro F et al (2013) Moderate exercise increases the metabolism and immune function of lymphocytes in rats. Eur J Appl Physiol 113(5):1343-1352

41. Chen $\mathrm{N}$ et al (2020) Epidemiological and clinical characteristics of 99 cases of 2019 novel coronavirus pneumonia in Wuhan, China: a descriptive study. Lancet 395(10223):507-513

42. Abd El-Kader SM, Al-Shreef FM (2018) Inflammatory cytokines and immune system modulation by aerobic versus resisted exercise training for elderly. Afr Health Sci 18(1):120-131

43. Falkenberg R, Eising C, Peters M (2018) Yoga and immune system functioning: a systematic review of randomized controlled trials. J Behav Med 41(4):467-482

44. Nieman DC, Wentz LM (2019) The compelling link between physical activity and the body's defense system. J Sport Health Sci 8(3):201-217

45. Campbell JP, Turner JE (2018) Debunking the myth of exerciseinduced immune suppression: redefining the impact of exercise on immunological health across the lifespan. Front Immunol 9:648

46. Campbell JP et al (2009) Acute exercise mobilises CD8+ T lymphocytes exhibiting an effector-memory phenotype. Brain Behav Immun 23(6):767-775

47. Martin SA, Pence BD, Woods JA (2009) Exercise and respiratory tract viral infections. Exerc Sport Sci Rev 37(4):157

48. Dinh HC et al (2017) Effects of physical exercise on markers of cellular immunosenescence: a systematic review. Calcif Tissue Int 100(2): 193-215

49. Campbell JP et al (2010) The effects of vaccine timing on the efficacy of an acute eccentric exercise intervention on the immune response to an influenza vaccine in young adults. Brain Behav Immun 24(2):236-242 
50. Jee Y-S (2019) Exercise is an antigen for vaccination: first series of scientific evidence. J Exerc Rehabil 15(3):339

51. Simpson RJ et al (2020) Can exercise affect immune function to increase susceptibility to infection? Exerc Immunol Rev 26:8-22

52. Hammami A et al (2020) Physical activity and coronavirus disease 2019 (COVID-19): specific recommendations for home-based physical training. Manag Sport Leis 27(12):1-6

53. Senna SM et al (2016) Moderate physical training attenuates perinatal low-protein-induced spleen lymphocyte apoptosis in endotoxemic adult offspring rats. Eur J Nutr 55(3):1113-1122

54. Bigley AB et al (2014) Acute exercise preferentially redeploys NK-cells with a highly-differentiated phenotype and augments cytotoxicity against lymphoma and multiple myeloma target cells. Brain Behav Immun 39:160-171
55. Guazzi M et al (2004) Improvement of alveolar-capillary membrane diffusing capacity with exercise training in chronic heart failure. J Appl Physiol 97(5):1866-1873

56. García I et al (2021) Lung capacity and alveolar gas diffusion in aquatic athletes: implications for performance and health. Apunts Sports Med 56(209):100339

57. Ahmad A et al (2013) A review on therapeutic potential of Nigella sativa: a miracle herb. Asian Pac J Trop Biomed 3(5):337-352

Publisher's Note Springer Nature remains neutral with regard to jurisdictional claims in published maps and institutional affiliations. 